\title{
Radio Observation of Coronal Mass Ejections (CMEs) Due to Flare Related Phenomenon on $7^{\text {th }}$ March 2012
}

\author{
Z. S. Hamidi ${ }^{1, *}$, C. Monstein ${ }^{2}$, N. N. M. Shariff ${ }^{3}$ \\ ${ }^{1}$ MARA University of Technology, 40450, Shah Alam, Selangor, Malaysia \\ ${ }^{2}$ Institute of Astronomy, Wolfgang-Pauli-Strasse 27, Building HIT, Floor J, \\ $\mathrm{CH}-8093$ Zurich, Switzerland \\ ${ }^{3}$ Academy of Contemporary Islamic Studies (ACIS), MARA University of Technology, \\ 40450, Shah Alam, Selangor, Malaysia \\ *E-mail address: zetysh@salam.uitm.edu.my
}

\begin{abstract}
On March 7th, 2012, an active region AR1429 has unleashed 2 major X-class solar flares. This flare is accompanied by a Coronal Mass Ejections (CMEs) event. A pair of unusually large solar flares early March 7, 2012 generated a Coronal Mass Ejection that was expected to reach Earth around midday March 8. In this case we focused on the second explosion of solar flare. It is found that the indication of signal potentially drives Coronal Mass Ejections (CMEs). There are a few types solar burst that can be observed, which is (i) an individual type III (ii) a complex type III (iii) subtype an $\mathrm{H}$ type II solar burst and (iv) type IV solar burst. The duration of solar burst is start from 1:02 UT to 2:00 UT. We also compare our results with the Geostationary Operational Environmental Satellites (GOES) data. Overall, one hour duration with a strong intensity burst are exploded strongly within the period. The fast drift type III burst has continued until 1:28 UT is associated with the large X 5.4 -class solar flares at 1:25:05 UT. It is undeniable that solar flare plays an important role in the Sun-Earth connection due to sudden changes of strong magnetic fields in the Sun's corona. From our analysis, one possible reason behind the formation of this very complex, long duration of this loop is the magnetic reconnection and disruption of the loops which is observed during flare maximum. Until now, there has been an increasing interest in the space weather program has stimulated interest in this issue. A new experimental approach by e-CALLISTO with 24 hours monitoring and further development of a model of the theory are hoping to meet the current knowledge about the Sun behaviour.
\end{abstract}

Keywords: Radio burst; solar flare; Coronal Mass Ejections (CMEs); e-CALLISTO; solar flare; Active Region 1429

\section{INTRODUCTION}

The radio observation has started since the observation of the Sun by Hey in 1944 [1]. One major finding in solar radio emission is the understanding of thermal radio emission of the large scale structure and physical state of the chromospheres and corona with a rapid transition between them from hot plasma [2]. 
This region is the widest region of the electromagnetic spectrum [3]. An extensive experimental and theoretical work has succeeded in elucidating many observational and characteristics of radio bursts in understanding their physical nature.

In the lower region, it can deliver an information on Sun activities such as Coronal Mass Ejections (CMEs) and solar flare. Solar radio observation plays an important role in understanding the mechanism of energy release, plasma heating, the particle acceleration and transfer in magnetized plasmas [4].

It is believed that the structure of the burst originates in the same layers of the solar atmosphere in which geo-effective disturbances probably originate the layers where energy is released in solar flares, where energetic particles are accelerated and where Coronal Mass Ejections (CMEs) are launched [5].

In their contribution, they have proved a strong correlation between bursts and solar flares and successfully determined two possible phases of particle acceleration. Therefore, it is important to study the dynamical process during the burst of flares [6,7].

A Coronal Mass Ejections (CMEs) related to the solar flare is very dominant in solar activities. Statistical study has shown that the CMEs events are mostly accompanied after a large solar flare event. However, what class of solar flare has a potential to form a CMEs still on-going study.

Solar flare is considered as a high energetic and complicated phenomenon in which mass eruptions occur, energetic particles are generated and highly energy radiations are emitted. It is undeniable that solar flare plays an important role in the Sun-Earth connection due to sudden changes of strong magnetic fields in the Sun's corona.

Within short time intervals of about $10^{2} \sim 10^{3} \mathrm{~s}$, large quantities of energy of $10^{22} \sim 10^{26}$ $\mathrm{J}$ are emancipated [8]. During a flares, large quantities of energy are transferred between the corona and chromosphere through thermal conduction, non-thermal particle beams, radiation transport and mass motions.

This event is triggered by fast drift of individual sunspot proper motion within the complex magnetic configuration due to instabilities of equilibrium of coronal magnetic field [9]. The solar flare is classified into 6 categories with is $\mathrm{A}, \mathrm{B}, \mathrm{C}, \mathrm{M}, \mathrm{X}$ and $\mathrm{Z}$ can be categorized as a low explosion of solar flare while $\mathrm{M}, \mathrm{X}$ and $\mathrm{Z}$ are the huge solar flare. Over the past twenty years, there are many theories and proposals for the particle acceleration processes involved in flares. However, the most popular classes of mechanisms for electrons are accelerated by an electric field parallel to the magnetic field by shocks and stochastic acceleration by waves [10].

This study concerned about solar flares and Coronal Mass Ejections. The solar flares and CMEs are usually accompanied by solar radio bursts and the signals are usually can be characterized by background levels of radiation upon which are superimposed bursts.

Solar flares and CMEs monitoring in radio region is very important in order to identify the active flares sources based on their nature and emission mechanisms and to relate their properties with plasma parameters in flaring regions [11]. These bursts originate by bremsstrahlung, gyrosynchrotron and plasma radiations.

\section{EXPERIMENTAL SETUP AND METHODOLOGY}

In this work, we used the Compound Astronomical Low-cost Low-frequency Instrument for Spectroscopy and Transportable Observatory (CALLISTO) data from SSRT site. There are several sites that successfully detected this flare. 
We also have the same system which is connected to the Log Periodic Dipole Antenna (LPDA) at the National Space Centre (ANGKASA), Selangor located at $\left(3.0833333^{\circ} \mathrm{N}\right.$ $101.5333333^{\circ} \mathrm{E}$ ) with minimum Radio Frequency Interference (RFI) noise level, with an average - (85-100) dBm [12-14]. The size of the antenna is 5.5 meters [15]. So far we have analyzed a few important solar events associated with solar burst type II [16], type III [17] and type IV $[18,19]$.

After a few month, we have modified the antenna with 3.3 meters [20]. In this network, we are focused from $45-870 \mathrm{MHz}$ where the LPDA or the telescope is connected to the CALLISTO spectrometer.

At our site, we focus only in the region of $260 \mathrm{MHz}$ till $380 \mathrm{MHz}$ [18]. The distribution data radio flux density in burst versus the frequency level has been collected daily starting from 11.30 UT till 23.30 UT [21-30].

\section{RESULTS AND ANALYSIS}

This event occurred on March 7th, 2012 in the Active Region 1429, located close to the limb of the solar disc, was recorded by the STEREO 2. In this case, we are choosing a data from SSRT site. This is one of the e-CALLISTO network that successfully detected the solar flare event. This site is one of the first sites that can be detected due to the period of the observation.

There are a few types solar burst that can be observed, which is (i) an individual type III (ii) a complex type III (iii) subtype H type II solar burst and (iv) type IV solar burst. The duration of solar burst is start from 1:02 UT to 2:00 UT.

Overall, one hour duration with a strong intensity burst are exploded strongly within the period. It is found that the indication of signal potentially drives Coronal Mass Ejections (CMEs). The chronology of the formation of solar burst associated with solar flare is presented from Figure 1 to Figure 5.

The first detection of a singular solar burst type III, and a group and complex solar burst type III with a very high intensity on 7th March 2012 detected at SSRT site from 00:50 UT1:06 UT. An individual solar burst type III can be seen at 00:57 UT in the region from $90-$ $320 \mathrm{MHz}$. After four minutes later, a group and very complex solar burst type III formed intermittently. The fast drift type III burst has continued until 1:28 UT is associated with the large X 5.4 -class solar flares at 1:25:05 UT.

We continue to analyze the event. In Figure 4, one could see, a broad solar burst type IV at 1:34 UT to 1:44 UT. It shows a strong signal from active region 1429. It should be noted that this active region is very active within a few days.

The burst is long lasting for a few minutes. Interestingly, an H-subtype type II solar burst can be observed at 1:58 UT. This is the last type of solar burst can be observed. H type solar burst is one of a sub type solar burst type II. 


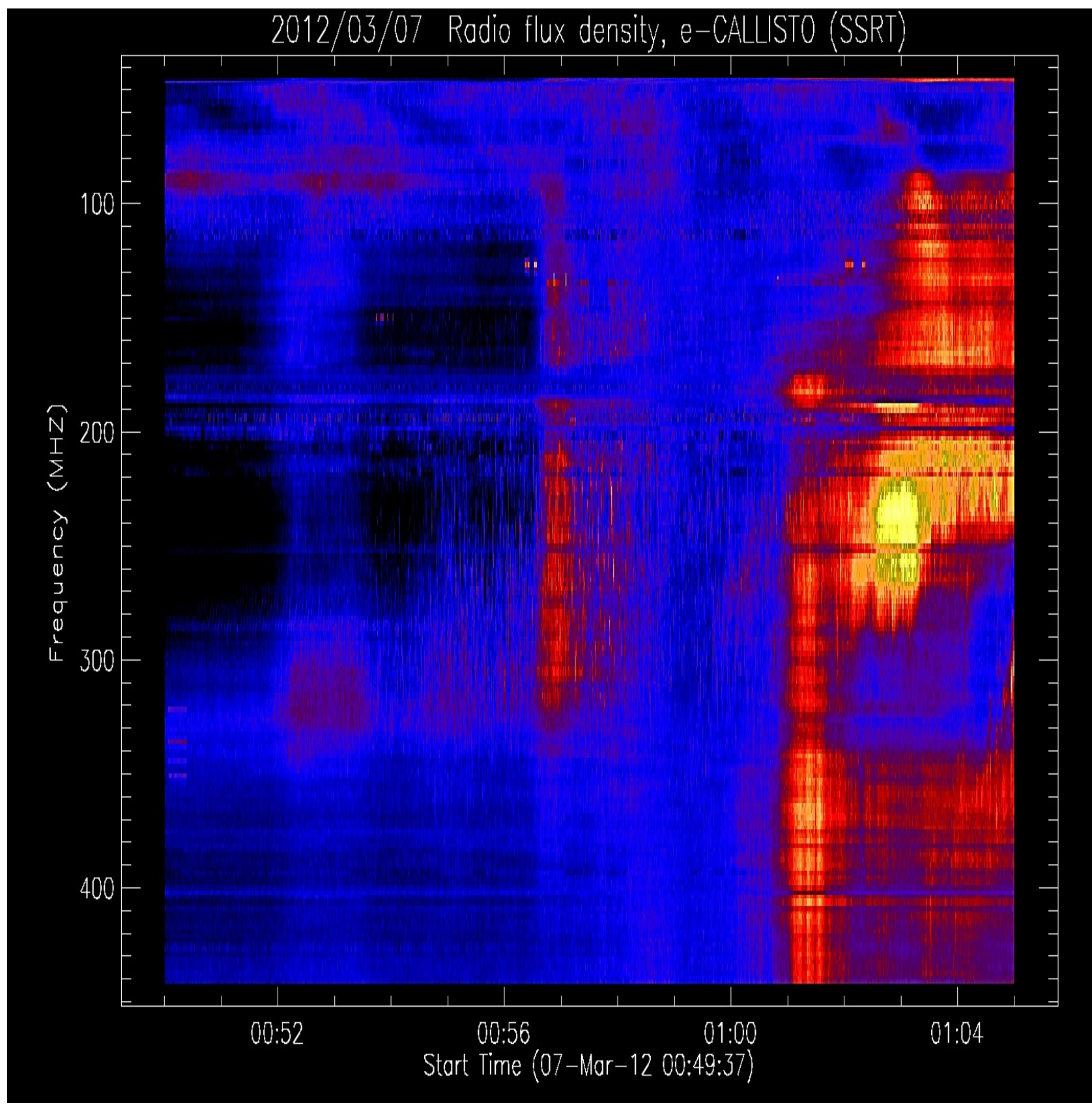

Figure 1. First detection of a singular solar burst type III, and a group and complex solar burst type III with a very high intensity on 7th March 2012 detected at SSRT site from 00:50 UT-1:06 UT. 


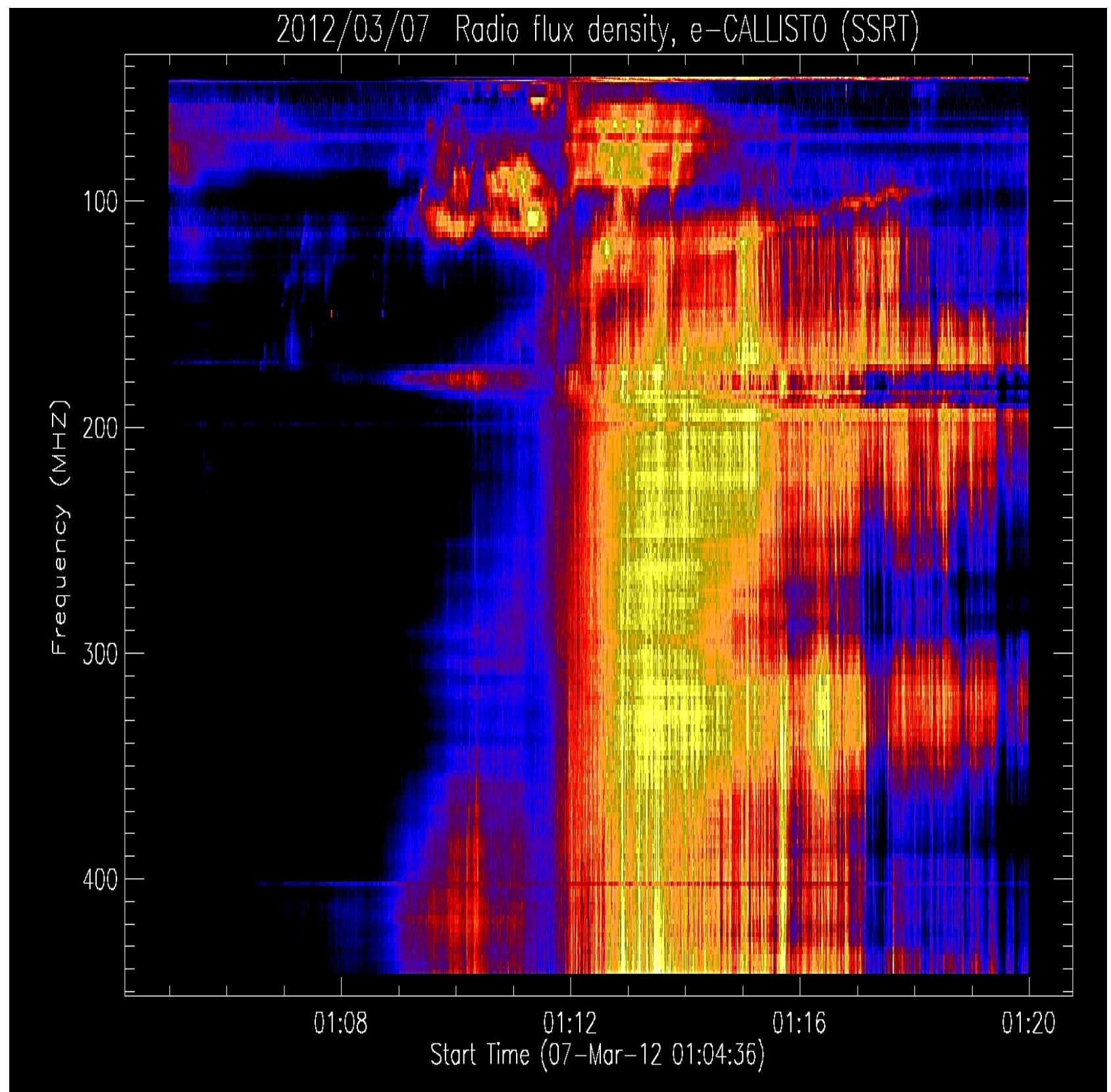

Figure 2. Continuous of a group and complex solar burst type III with a very high intensity on 7th March 2012 detected at SSRT site from 1:06 UT-1:20 UT. 


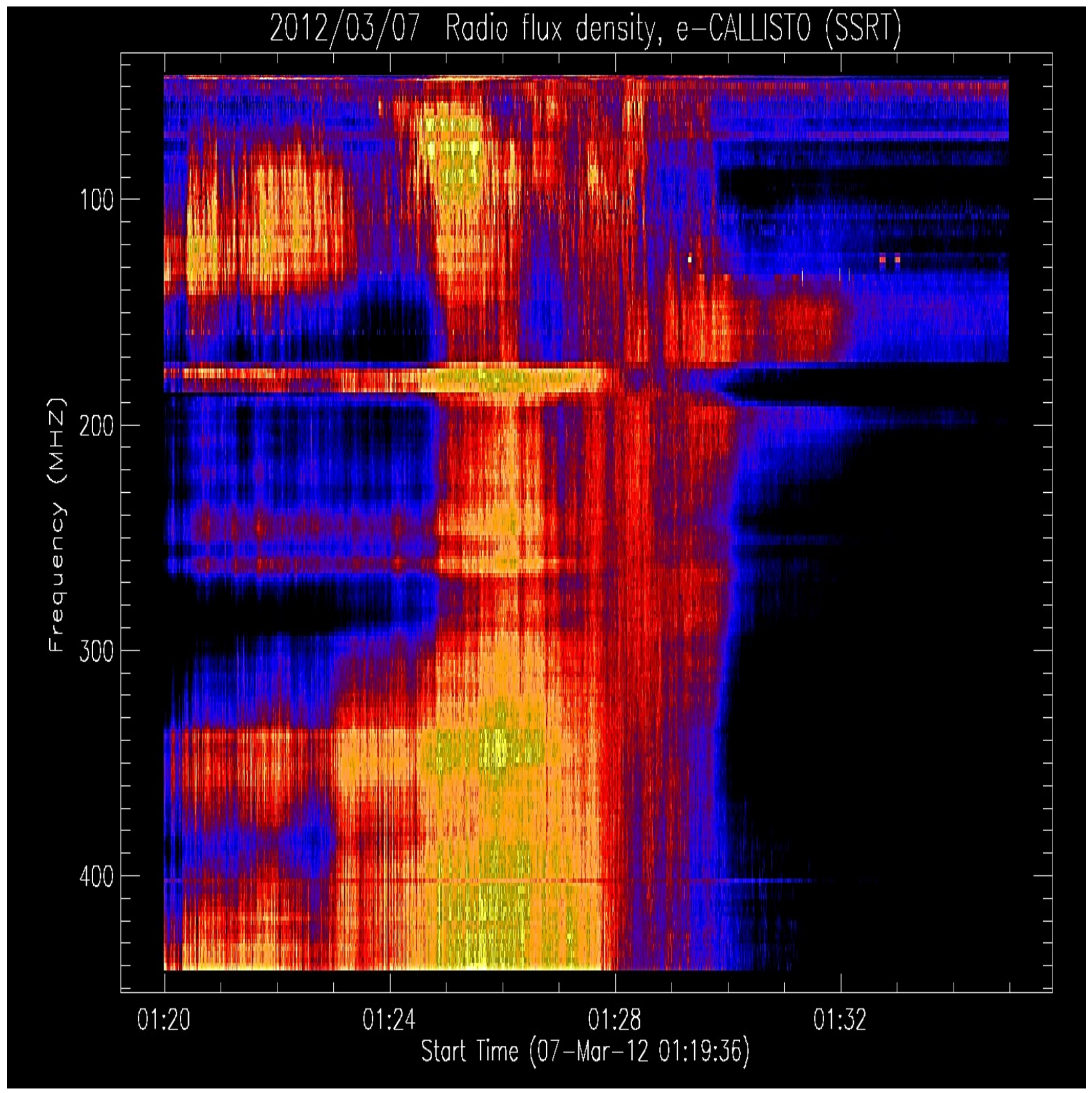

Figure 3. Continuous of a group and complex solar burst type III with a very high intensity on 7 th March 2012 detected at SSRT site from 1:20 UT-1:34 UT. 


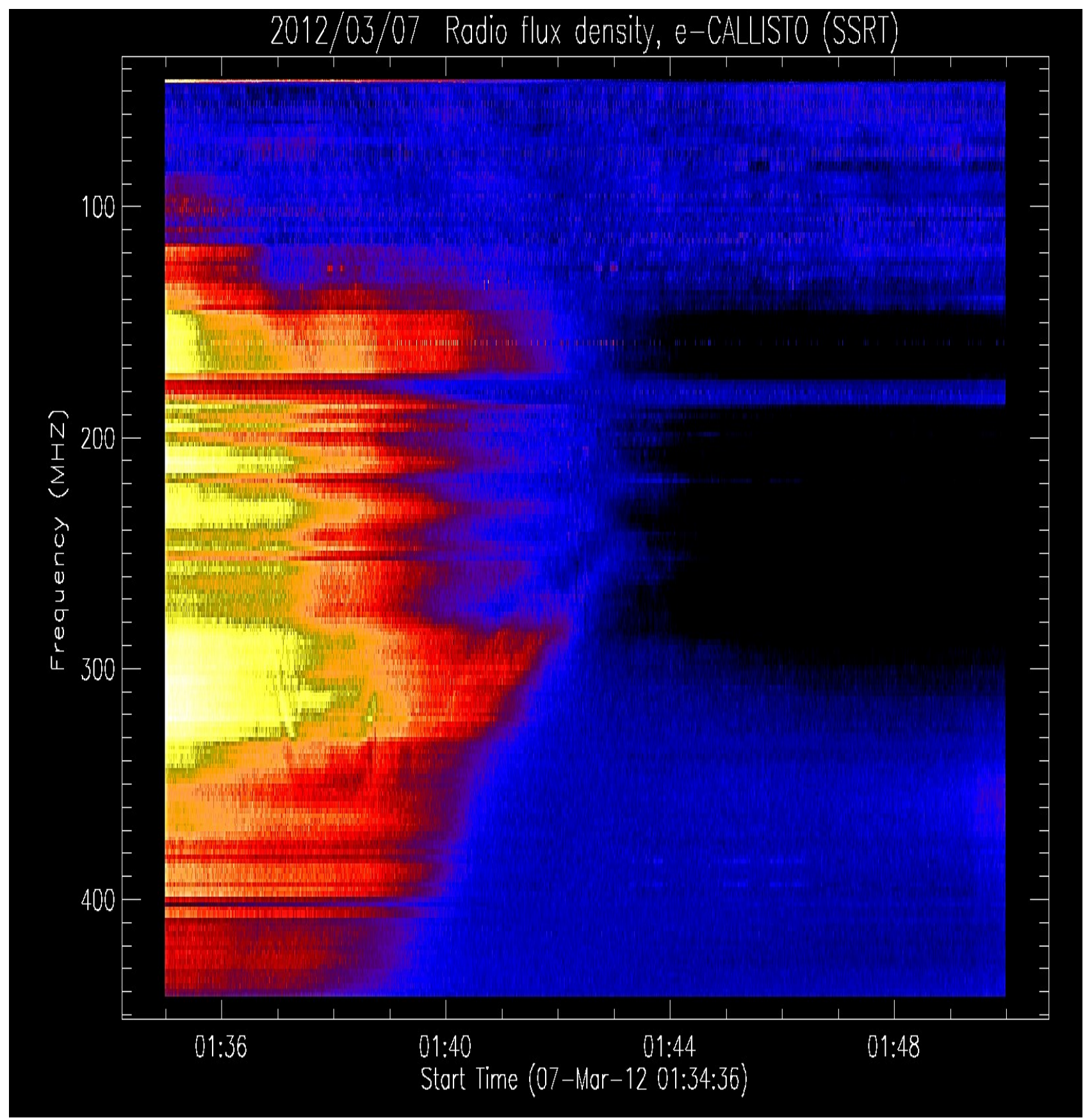

Figure 4. A broad type IV solar burst with a very high intensity on 7th March 2012 detected at SSRT site from 1:34 UT-1:50 UT. 


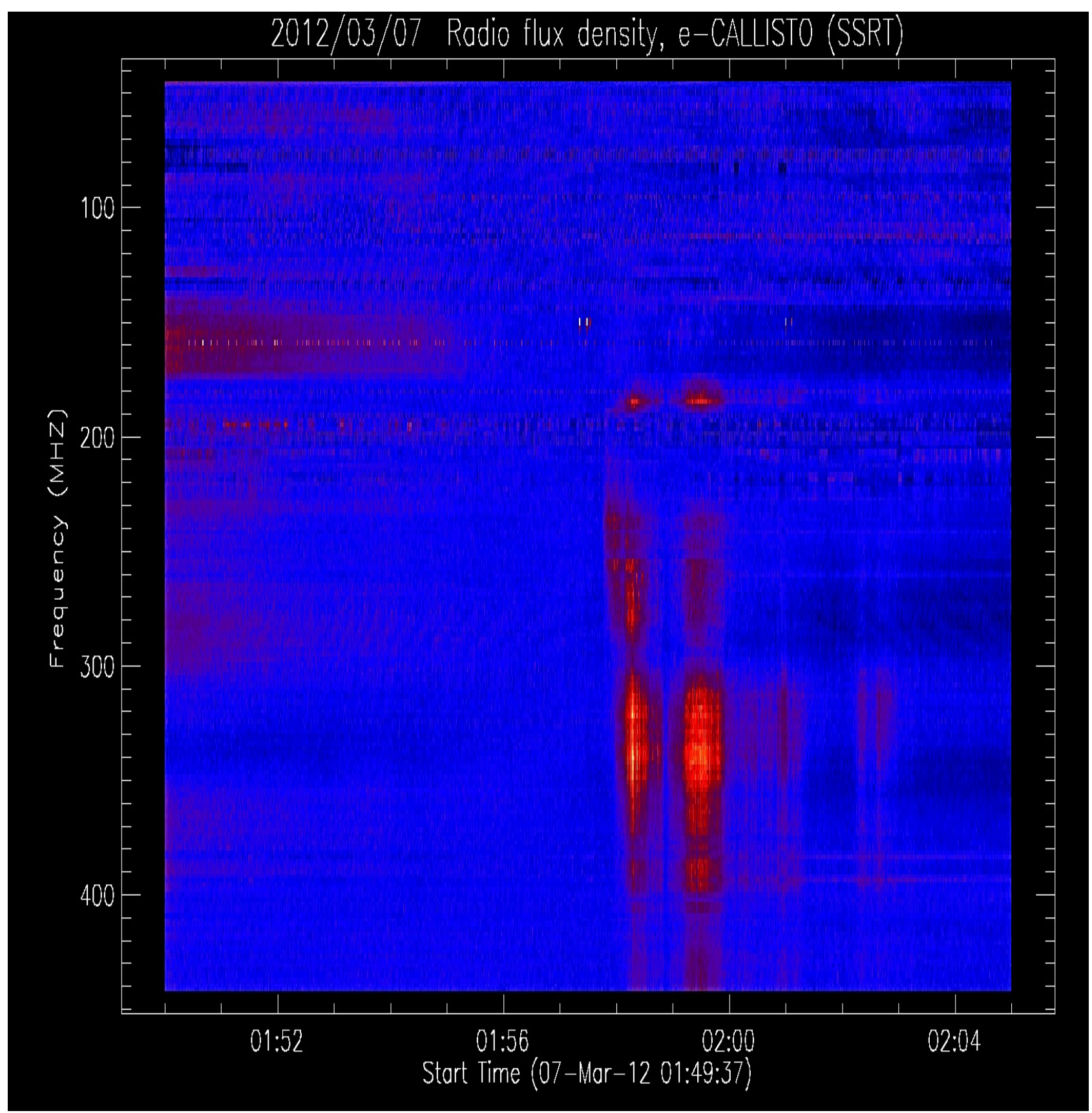

Figure 5. An H-subtype type II solar burst can be observed at 1:58 UT on 7th March 2012 detected at SSRT site from 1:50 UT-2:05 UT.

During this stage, the Sun's radio emission can increase up to a million times the normal intensity just a few seconds. A $10 \mathrm{~cm}$ radio burst indicates that the electromagnetic burst associated with a solar flare at the $10 \mathrm{~cm}$ wavelength was double or greater than the initial 10 $\mathrm{cm}$ radio background. From the observations, strong bursts that caused by extraordinary solar flares due to magnetic reconnection effect potentially induced in the near-Earth magneto tail. One could see the tenuous plasma in that region is then accelerated down magnetic field lines into the Polar Regions, striking Earth's atmosphere and exciting nitrogen and oxygen atoms as well as the other atoms present in our atmosphere. Theoretically, time variability in the emission may due to the changes in the electron density. Thus, this active region has remained 
active in a few days. It is expected that it will be larger solar flares and CMEs in the next few days. Fortunately, this explosion is not directed in the direction to the Earth. The Active Region 1429 poses a threat for the X 5.4 -class solar flares at 1:25:05 UT in Figure 6.

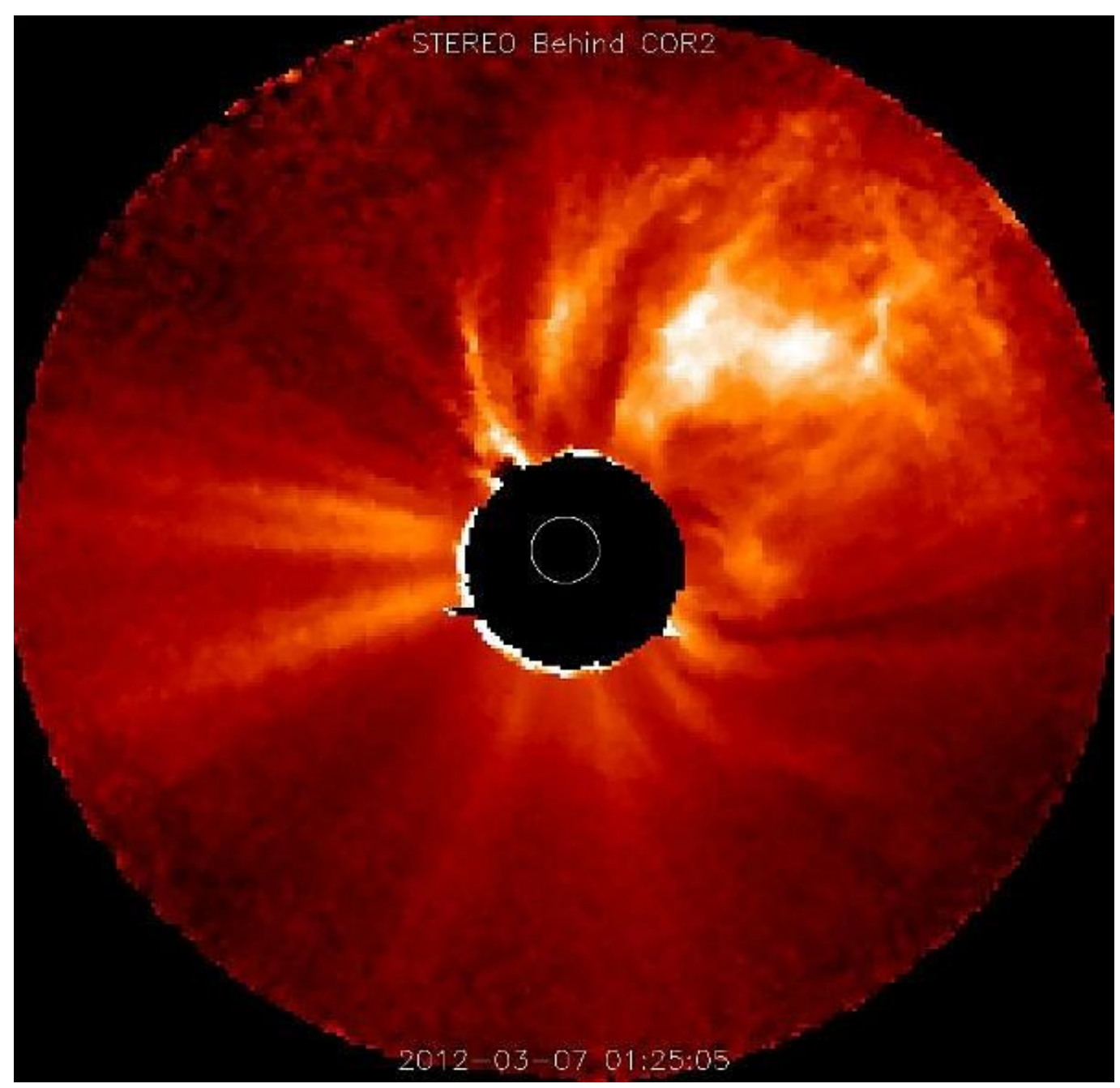

Figure 6. The Active Region 1429 poses a threat for the X 5.4 - class solar flares at 1:25:05 UT. Credited to: STEREO2

It is believed that the changing magnetic field converts magnetic potential energy into kinetic energy by accelerating plasmas in the corona. In order to confirm our results, we used a STEREO2 data. It was found that this flare has triggered a strong R3 Level Radio Blackout. Soon after that, a CMEs explosion occurs due to the active region 1429. The solar wind is showing a sudden increase from $360 \mathrm{kms}^{-1}$ to $440 \mathrm{kms}^{-1}$ and the IMF is currently tilting sharply south. There will be a chance for some sort of impact. The X-ray flux from GOES from 5th - 7th March 2012 is shown in Figure 7. 


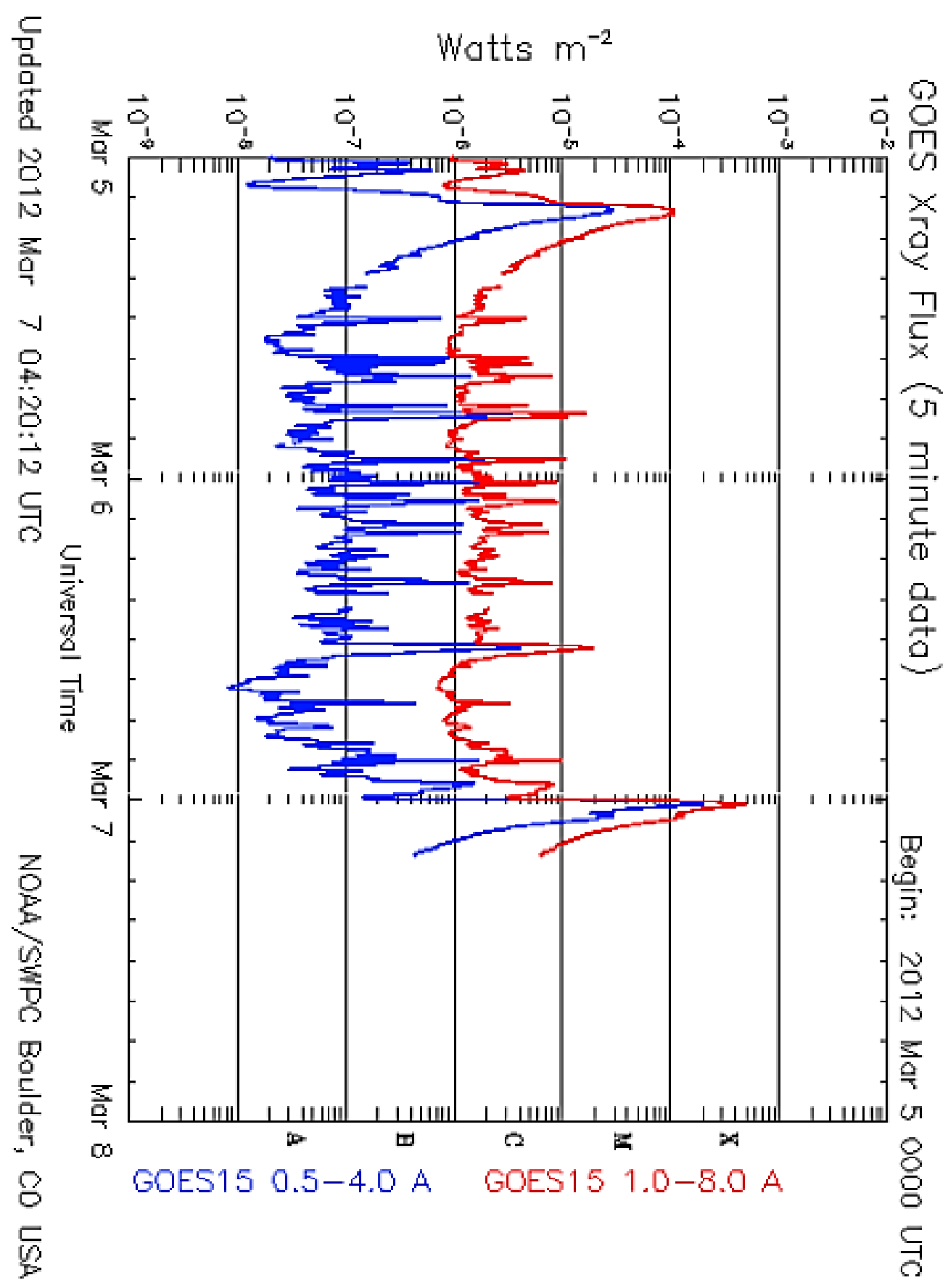

Figure 7. The X-ray flux from GOES from $5^{\text {th }}-7^{\text {th }}$ March 2012. Credited to: GOES.

Based on the analysis, it is believed that the formation of this flare is very complex and take a long duration before a large flare of class $\mathrm{X}$ is exploded. The extra-large two solar flares occurred at 23:24 GMT on 6 March and 00:14 GMT March 7, flinging off a huge cloud of magnetized plasma from the Sun's atmosphere (solar corona) into interplanetary space.The loop of the structure of the flare proved a magnetic reconnection and disruption of the loops which is observed during flare maximum. 


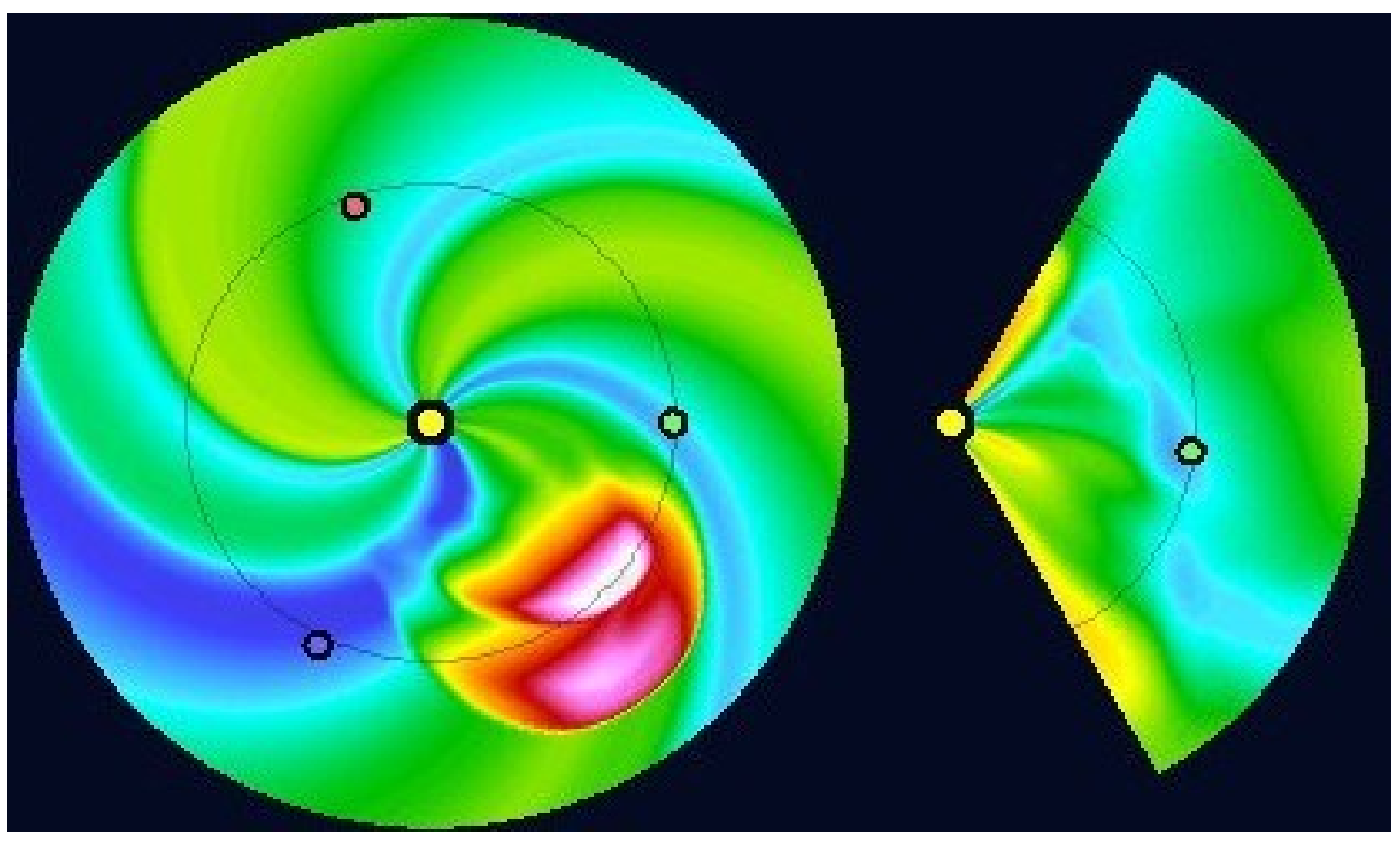

Figure 8. The bright Coronal Mass Ejection (CMEs) position from STEREO.

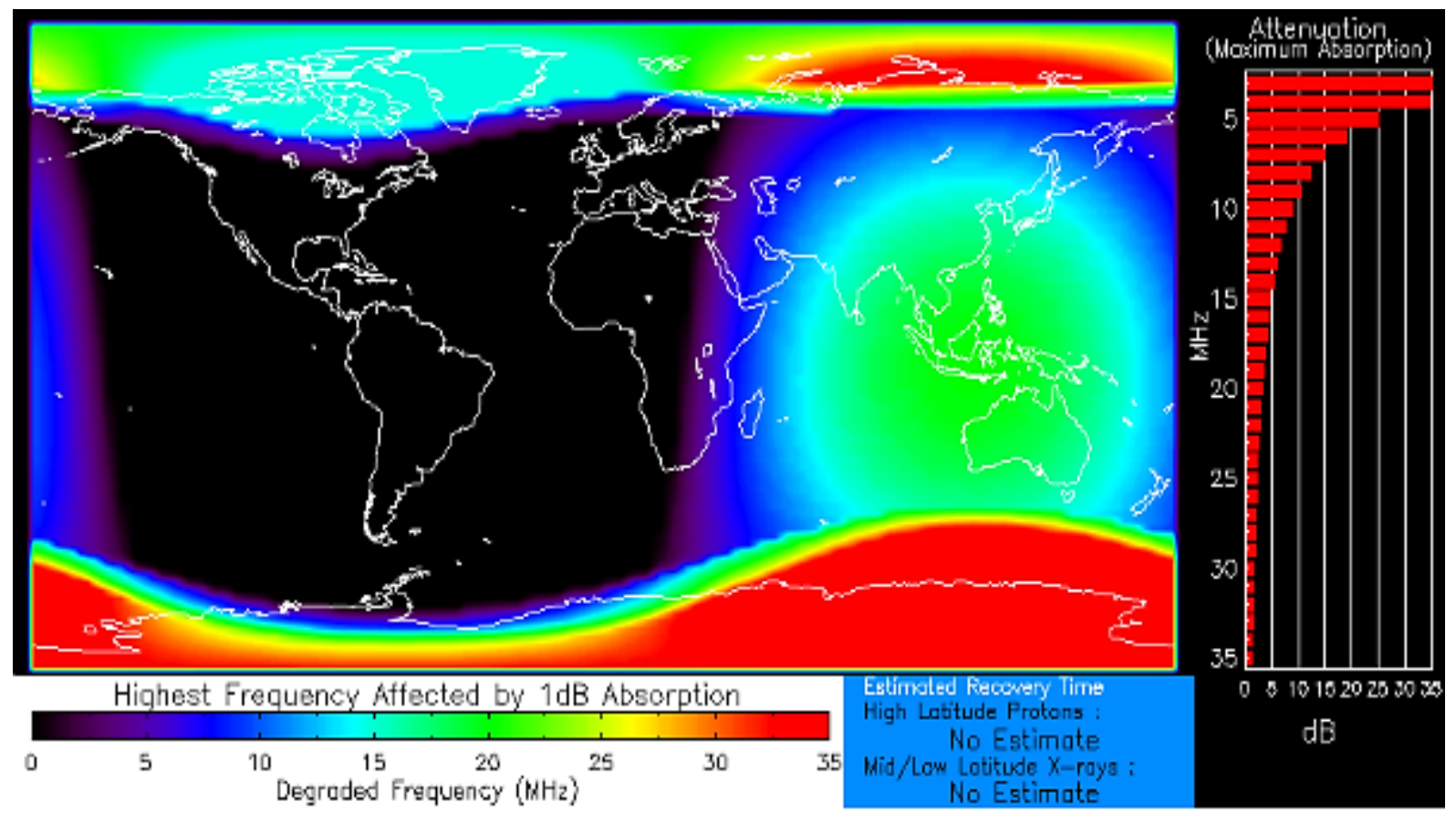

Elevated $x$-ray flux

Product Volid At : 2012-03-07 04:21 UTC

Normal Proton Background

NOAA/SWPC Boulder, CO USA

Figure 9. The elevated of X-ray flux at the Earth (Credited: SWPC/ NOAA). 
We could observe the Aurora phenomenon at high latitudes. In Figure 8, the bright Coronal Mass Ejection (CME) is seen in the new STEREO Behind COR2 images. The high energy proton levels are now on the rise and a radiation storm is imminent. Solar burst type II emissions occur in association with eruptions on the sun and typically indicate a coronal mass ejection is associated with a flare event.

The impact of Coronal Mass Ejections (CMEs) to the Earth with the aurora phenomena is illustrated in Figure 9.

\section{CONCLUSION}

Radio observations combined with the Hard X-ray (HXR) observations can help us to analyze the release of flare energy and acceleration of energetic particles during the pre-phase of the flare. The burst is originated in the same layers of the solar corona in which geoeffective disturbance probably initiates. A pair of unusually large solar flares early March 7, 2012 generated a Coronal Mass Ejection that was expected to reach Earth around mid-day March 8.

This flare is considered one of the largest solar flare in 2012. Until now, there has been an increasing interest in the space weather program has stimulated interest in this issue. A new experiment approach by e-CALLISTO with 24 hours monitoring and further development of a model of the theory are hoping to meet the current knowledge about the Sun behaviour.

\section{ACKNOWLEDGEMENT}

We are grateful to SSRT (CALLISTO network), STEREO, LASCO,SDO/AIA, NOAA and SWPC make their data available online. This work was partially supported by the FRGS (600 RMI/FRGS 5/3 2012) UiTM grants. Special thanks to the National Space Agency and the National Space Centre for giving us a site to set up this project and support this project. Solar burst monitoring is a project of cooperation between the Institute of Astronomy, ETH Zurich, and FHNW Windisch, Switzerland, MARA University of Technology and University of Malaya. This paper also used NOAA Space Weather Prediction Centre (SWPC) for the sunspot, radio flux and solar flare data for comparison purpose. The research has made use of the National Space Centre Facility and a part of an initiative of the International Space Weather Initiative (ISWI) program.

\section{BIOGRAPHY}

Dr Zety Sharizat Hamidi is currently a lecturer and focused in Solar Astrophysics research specifically in radio astrophysics at the School of Physics and Material Sciences, Faculty of Sciences, MARA University of Technology, 40450, Shah Alam, Selangor, Malaysia. Involve a project under the International Space Weather Initiative (ISWI) and also a lecturer in School of Physics and Material Science, at MARA University of Technology, Shah Alam Selangor.

C.Monstein is a senior Engineer at Institute of Astronomy, Wolfgang-Pauli-Strasse 27, Building HIT, Floor J, CH-8093 Zurich, Switzerland and one of the researchers who initiated the CALLISTO system around the world.

Dr Nur Nafhatun Md Shariff is a senior lecturer in Academy of Contemporary Islamic Studies (ACIS), MARA University of Technology, 40450, Shah Alam, Selangor, MalaysiaHer current research is more on sustainability; environmental aspect. She is looking forward for cross-field research, i.e. solar astrophysics, light pollution measurement (mapping) and religious studies. 


\section{References}

[1] J.S. Hey, S.J. Parsons, J.W. Phillips, Monthly Notices of the Royal Astronomical Society 108 (1948) 354-371.

[2] A.O. Benz, Flare Observations, Living Rev. Solar Phys. 5 (2008).

[3] Z.S. Hamidi, N.N.M. Shariff, C. Monstein, Z. Abidin, Z. Ibrahim, N. Hashim, R. Umar, N. Aziz, International Journal of Fundamental Physical Sciences 3 (2013) 57-63.

[4] M.R. Kundu, Solar Radio Astronomy, John Wiley, 1965.

[5] S.M. White, Solar Radio Bursts and Space Weather, Asian Journal of Physics 16 (2007).

[6] T.S. Bastian, Benz, A.O., Gary, D.E., Radio emission from solar flares, Annu. Rev. Astron. Astrophys. 36 (1998).

[7] M. Aschwanden, Physics of the Solar Corona, Praxis Publication, Chichester, UK, 2004.

[8] G.W. Pneuman, Case of Hydromagnetic Resonance 2 (1967) 462-483.

[9] D. Herdiwijaya, S. Imelda, Jurnal Matematika dan Sains 11 (2006) 7.

[10] J.A. Miller, P. J. Cargill, G. Emslie, J. Geophys. Res. (1997) 4631-4660.

[11] V.V. Grechnev, An Impulsive Solar Flare Accompanied by a Cusplike Structure in Soft X-Rays, ApJ 566 (2002).

[12] Z.S. Hamidi, Z. Ibrahim, Z. Abidin, M. Maulud, N. Radzin, N. Hamzan, N. Anim, N. Shariff, International Journal of Applied Physics and Mathematics 2 (2011) 3.

[13] Z.S.Hamidi, et al., International Journal of Fundamental Physical Sciences 2 (2012) 4.

[14] Z.S.Hamidi, N.N.M.Shariff, R.Umar, Malaysia Thailand Journal of Physics 3 (2012) 6.

[15] Z.S. Hamidi, Z. Ibrahim, Z. Abidin, M. Maulud, N. Radzin, N. Hamzan, N. Anim, N. Shariff, International Journal of Applied Physics and Mathematics 2 (2012) 140-142.

[16] Z.S.Hamidi, Z. Abidin, Z. Ibrahim, N. Shariff, C. Monstein, Observations of coronal mass ejections (CMEs) at low frequency radio region on 15th April 2012, in: R.Shukor (Ed.), PERFIK 2012, American Institute of Physics, Malaysia, 2013, pp. 5.

[17] Z.S. Hamidi, N. Anim, N.N.M. Shariff, Z.Z. Abidin, Z.A. Ibrahim, C. Monstein, Dynamical structure of solar radio burst type III as evidence of energy of solar flares, in: R.Shukor (Ed.), PERFIK 2012, American Institute of Physics, Malaysia, 2013, pp. 11-15.

[18] Z.S.Hamidi, Z. Abidin, Z. Ibrahim, C. Monstein, N. Shariff, International Journal of Fundamental Physical Sciences 2 (2012) 32-34.

[19] Z.S. Hamidi, N.N.M. Shariff, F.N.Z. Ulum, Z.Z. Abidin, Z.A. Ibrahim, International Journal of Astronomy 5 (2012) 101-106.

[20] Z.S. Hamidi, C. Monstein, Z.Z. Abidin, Z.A. Ibrahim, N.N.M. Shariff, International Journal of Engineering Research and Development 3 (2012) 36-39.

[21] Z.S. Hamidi, N. Shariff, Z. Abidin, Z. Ibrahim, C. Monstein, Middle-East Journal of Scientific Research 12 (2012) 6. 
[22] Z.S.Hamidi, N.N.M.Shariff, Evaluation of Signal to Noise Ratio (SNR) of Log Periodic Dipole Antenna (LPDA) Business Engineering and Industrial Applications Colloquium 2013, IEEE, Langkawi, Malaysia, 2013, pp. 434-438.

[23] Z.S. Hamidi, N.N.M.Shariff, International Letters of Chemistry, Physics and Astronomy 4 (2014) 29-36.

[24] Z.S. Hamidi, N.N.M. Shariff, M.F. Ali, C. Monstein, W.N.A.W. Zulkifli, M.B. Ibrahim, N.S. Arifin, N.A. Amran, International Letters of Chemistry, Physics and Astronomy 9 (2014) 84-92.

[25] Z.S. Hamidi, N.N.M.Shariff, C.Monstein, W.N.A.W. Zulkifli, M.B. Ibrahim, N.S. Arifin, N.A. Amran, International Letters of Chemistry, Physics and Astronomy 8 (2014) 13-19.

[26] Z.S. Hamidi, N.N.M.Shariff, International Letters of Chemistry, Physics and Astronomy 7 (2014) 30-36.

[27] Z.S. Hamidi, N.N.M.Shariff, International Letters of Chemistry, Physics and Astronomy 5 (2014) 32-42.

[28] Z.S. Hamidi, N.N.M.Shariff, C.Monstein, Z.A. Ibrahim, International Letters of Chemistry, Physics and Astronomy 7 (2014) 37-44.

[29] Z. S. Hamidi, N. N. M. Shariff, C. Monstein, International Letters of Chemistry, Physics and Astronomy 11(1) (2014) 51-58.

[30] Z. S. Hamidi, N. N. M. Shariff, C. Monstein, International Letters of Chemistry, Physics and Astronomy 11(2) (2014) 135-145. 